\title{
Biolixiviación microbiana del cobre como tecnología para la explotación del mineral microbial
}

Microbial bioleaching of copper as a technology for the exploitation of microbial mineral

Recibido: noviembre 12 del 2018 | Revisado: diciembre 18 del 2018 | Aceptado: febrero 12 del 2019

Paola del Rosario Eyzaguirre Liendo ${ }^{\mathrm{I}}$ Daladier Miguel Castillo Cotrina²

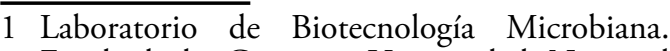
Facultad de Ciencias. Universidad Nacional Jorge Basadre Grohmann (UNJBG). Tacna, Perú.

paolaeyzaguirre41@gmail.com

daladiercastillo@hotmail.com

\begin{abstract}
Resumen
La búsqueda de alternativas tecnológicas sostenibles para el drenaje ácido de minas es una necesidad. El propósito del estudio fue analizar de forma exploratoria la biolixiviación del cobre como tecnología de extracción del mineral. Desde el 2014 hasta el 2015 se determinó la producción promedio máxima microbiana de lixiviados pertenecientes a cuatro botaderos del asiento minero de Toquepala pertenecientes a la Empresa Southern Perú Coper. Las concentraciones promedio con relación a la determinación de cobre $(0,5,10,15,20)$ fueron: 8,903089987 $\pm 0,026 ; 7,778151250 \pm 0,058,7,204119983$ $\pm 0,071,7,041392685 \pm 0,039$ y 7,000000000 $\pm 0,016$ donde se encontró diferencias estadísticamente significativas $(\mathrm{p}<0,05)$. Los resultados experimentales indicaron que, el cobre puede extraerse mediante el proceso de biolixiviación.
\end{abstract}

Palabras clave: drenaje ácido, biolixiviación, cobre, extracción mineral

\begin{abstract}
The search for sustainable technological alternatives for acid mine drainage it is a necessity. The purpose of study was to explore exploratoryly the bioleaching of copper as a mineral extraction technology. From 2014 to 2015, the maximum average microbial production of leachates belonging to four mining dumps of Toquepala belonging to Southern Peru Copper Company was determined. The average concentrations in relation to the determination of copper $(0,5,10,15,20)$ were: 8,903089987 $\pm 0,026 ; 7,778151250 \pm 0,058,7,204119983 \pm$ $0,071,7,041392685 \pm 0.039$ and 7,000000000
\end{abstract}

(C) Los autores. Este artículo es publicado por la Revista Campus de la Facultad de Ingeniería y Arquitectura de la Universidad de San Martín de Porres. Este artículo se distribuye en los términos de la Licencia Creative Commons Atribución No-comercial - Compartir-Igual 4.0 Internacional (https://creativecommons.org/licenses/ CC-BY), que permite el uso no comercial, distribución y reproducción en cualquier medio siempre que la obra original sea debidamente citada. Para uso comercial contactar a: revistacampus@usmp.pe. 
$\pm 0,016$ where statistically significant differences were found $(p<0,05)$. The experimental results indicated that copper can be extracted through the bioleaching process.

Key words: acid drainage, bioleaching, copper, mineral extraction

\section{Introducción}

La exposición a los relaves (Nejeschlebová et al., 2015) y remoción de tierra para la extracción de elementos metálicos como el cobre ocasiona contaminación ambiental (Amari et al., 2014; Beylot \& Villeneuve, 2017; Kefeni et al., 2017). El cobre es un elemento no ferroso muy costoso y altamente demandado a nivel mundial (Volchko et al., 2017), cuyas reservas podrían agotarse para el 2050 (Brown, 2006).

El cobre, una vez impregnado en aquellos materiales catalogados como contaminados, puede recuperarse mediante técnicas $e x$-situ de lavado químico (Karlfeldt, Yillin \& Strömvall, 2013). La lixiviación es una de las técnicas de recuperación (Adedigba, 2015; Andersson \& Lundström, 2015) existiendo bacterias asociadas durante el proceso (Kondrat'eva et al., 2012; Hao et al., 2016) las cuales, a través de tecnologías genéticas son identificadas (Korehi, Blothe \& Schippers, 2014; Gupta et al., 2017). Con la finalidad de proteger el entorno ambiental, tecnologías como la biolixiviación se aplican de forma sistemática (Johnson, 2014; Harrison, 2016; Cox \& Bryan, 2017; Yin et al., 2018) y en particular, en aquellos concentrados de cobre (Brierley, 2016).

Desde la década de 1940, la biosolubilización de óxidos y sulfuros metálicos (Mishra et al., 2005) en la minería de cobre se practican en varios países con fines comerciales (Ehrlich, 2001). Durante el proceso de lixiviación diversas especies se encuentran involucradas como por ejemplo: Acidithiobacillus ferrooxidans, Acidithiobacillus thiooxidans, Acidithiobacillus caldus, Acidithiobacillus albertis, Acidithiobacillus acidophilus, Acidithiobacillus concretivorus, Acidithiobacillus prosperus (Hoque \& Philip, 2011) y Leptospirillum ferrooxidans (Ewart \& Martin, 1991; Bosecker, 1997; Rawlings, 2013). La inmovilización con Acidithiobacillus ferrooxidans, oxidan un sustrato que contiene el hierro ferroso $\left(\mathrm{Fe}^{2+}\right)$ para formar compuestos de hierro férrico $\left(\mathrm{Fe}^{3+}\right)$ en solución altamente ácida y cada vez más, está siendo utilizada para la biooxidación de hierro $\mathrm{Fe}^{2+}$ (Mazuelos et al., 1999; Giaveno et al., 2008).

El cobre al ser un elemento esencial para los seres vivos (Taiz \& Zeiger, 2010) debido a la participación en múltiples procesos redox, formación del grupo prostético de algunas enzimas y en el transporte de electrones durante el proceso de la fotosíntesis, además de la respiración celular (Fernández \& Henríquez, 1991; Maksymiec, 1997; Yruela, 2005), es que su interés de explotación es reconocido. El propósito del estudio fue analizar la biolixiviación del cobre como tecnología de extracción del mineral.

\section{Desarrollo}

El drenaje ácido durante los procesos biolixiviantes, por lo general, tiene im- 
pactos ambientales negativos (Vahidi \& Zhao, 2016; Jones \& Johnson, 2016; Bonilla et al., 2018) por cuanto, la aplicación de procesos biotecnológicos resulta de interés para la extracción de minerales (Watling, 2016).

A temperatura y presión atmosférica relativamente baja los procesos de biolixiviación son funcionales y sostenibles debido a la reducción del costo energético, baja emanación de gases y limitada dependencia sobre reactivos costosos y agresivos (Bryan et al., 2015).

Aunque la biolixiviación no es $100 \%$ eficiente para la recuperación de mineral(Simate \& Ndlovu, 2014), al menos permite remediar en parte, el impacto zonal de la actividad minera (Park et al., 2015). A partir de la selección sobre cuatro botaderos que presentaron lixiviados del mineral calcopirita de baja ley, ubicados en el asiento minero de Toquepala y pertenecientes a la Empresa Southern Perú Cooper, fueron analizadas desde julio (2014) hasta julio (2015) diversas muestras en el Laboratorio de Biotecnología Microbiana de la Facultad de Ingeniería Metalúrgica perteneciente a la Universidad Nacional Jorge Basadre Grohmman, Tacna-Perú.
Una vez muestreadas la masa mineral de interés se sumergieron en $3 \mathrm{~L}$ de medio 9K (líquido modificado) a $\mathrm{pH} 1,8$ donde se insufló, $1 \mathrm{vvm}$ de aire durante 15 días a temperatura ambiente $\left(25^{\circ} \mathrm{C}\right)$ con agitación diaria (biorreactor). Se sembró mediante asa de Koll por extensión, inóculos tomados del cultivo de enriquecimiento sobre la superficie del medio de cultivo agar, $9 \mathrm{~K}$ contenido en placas Petri las cuales fueron incubadas a temperatura ambiente por 15 días para su observación al microscopio.

Para la obtención del inóculo (consorcio microbiano) se tomó $0,5 \mathrm{~L}$ de cultivo enriquecido por cultivo de enriquecimiento; y por separado se agregó con 3L de medio 9K líquido, $\mathrm{pH} \mathrm{1.8,} \mathrm{conteni-}$ do en un biorreactor que se agitó diariamente por 15 minutos, durante 15 días a temperatura ambiente con una aireación de 1 vvm.

La Tabla 1 muestra la concentración promedio de cobre y la producción promedio máxima microbiana donde se encontró diferencias estadísticamente significativas $(\mathrm{p}<0,05)$ en esta última variable (Tabla 2).

\section{Tabla 1}

Concentración de cobre / producción máxima microbiana

\begin{tabular}{cc}
\hline $\begin{array}{c}\text { Concentración de } \\
\text { cobre }(\mathrm{ppm})\end{array}$ & $\begin{array}{c}\text { Producción máxima microbiana } \\
(\text { logaritmo de cel/ml) }\end{array}$ \\
\hline 0 & $8,903089987(1)$ \\
5 & $7,778151250(2)$ \\
10 & $7,204119983(3)$ \\
15 & $7,041392685(4)$ \\
20 & $7,000000000(5)$ \\
\hline
\end{tabular}


Tabla 2

Análisis de la varianza

\begin{tabular}{|c|c|c|c|c|c|}
\hline FV & $\mathrm{SC}$ & $\mathrm{gl}$ & $\mathrm{CM}$ & Coeficiente $\mathrm{F}$ & Valor P \\
\hline Entre grupos & 7,6717 & 4 & 1,91792 & 239142713,20 & 0,0000 \\
\hline Intra grupos & $8,02 \mathrm{E}-8$ & 10 & $8,02 \mathrm{E}-9$ & & \\
\hline Total (Corr.) & 7,6717 & 14 & & & \\
\hline \multicolumn{3}{|c|}{ Prueba de Bonferroni } & & & \\
\hline $\begin{array}{c}\text { Producción máxima } \\
\text { microbiana }\end{array}$ & Grupc & hogéneos & & & \\
\hline 5 & & & & & \\
\hline 4 & & & & & \\
\hline 3 & & & & & \\
\hline 2 & & & & & \\
\hline 1 & & & & & \\
\hline
\end{tabular}

Según el estudio con enfoque cuantitativo y estado actual del conocimiento exploratorio, hubo posibilidad de extraer mediante procesos biolixiviación el cobre donde la producción máxima microbiana estuvo en función a la concentración de dicho elemento. Asimismo, para estudios futuros, se requiere establecer un diseño de experimento donde se deter- minen reacciones redox que puedan influir en la optimización del crecimiento microbiano.

Se concluyó que el mineral puede recuperarse siendo rentable en aquellos sitios donde existe exposición contaminante y para ello, el proceso biotecnológico por biolixiviación lo permitió.

\section{Referencias}

Adedigba, A. (2015). Assessment of Metal Recycling in Remediation Projects: Application and Evaluation of a Cost-benefit Analysis Method. (Master's Thesis No 2015:135). Chalmers Reproservice, Gothenburg. http://publications.lib.chalmers.se/records/fulltext/226582/226582.pdf

Amari, K.E., Valera, P., Hibti, M., Pretti, S., Marcello, A. \& Essarraj, S. (2014). Impact of mine tailings on surrounding soils and ground water: Case of Kettara old mine, Morocco. Journal of African Earth
Sciences; 100, 437-449. http:// dx.doi.org/10.1016/j.jafrearsci.2014.07.017

Andersson, D. \& Lundström, J. (2015). Enhanced Soil Washing - A Study on Treatment of Copper Polluted Soil and Bark. (Master's thesis 2015:45). Chalmers Reproservice, Gothenburg

Beylot, A. \& Villeneuve, J., 2017. Accounting for the environmental impacts of sulfidic tailings storage in the Life Cycle Assessment of copper production: A case study. Journal of 
Cleaner Production; 152, 139-145. http://dx.doi.org/10.1016\%2Fj. jclepro.2017.03.129

Bosecker, K. (1997). Bioleaching: metal solubilization by microorganisms - Bosecker - 1997. FEMS Microbiol. Rev; 20, 591-604. https://doi.org/10.1016/S01686445(97)00036-3

Brierley, C.L. (2016). Biological processing of sulfidic ores and concentrates - integrating innovations. In: Lakshmanan, V.I., Roy, R., Ramachandran, V. (Eds.): Innovative Process Development in Metallurgical Industry. Springer International Publishing, Switzerland; 109-135.

Brown, L., (2006). Plan B 2.0: rescuing a planet under stress and a civilization in trouble. Earth Policy Institute. W.W. Norton \& Co., New York. ISBN: 0-393-32831-7

Bryan, C., Watkin, E., McCredden, T., Wong, Z., Harrison, S. \& Kaksonen, A. (2015). The use of pyrite as a source of lixiviant in the bioleaching of electronic waste. Hydrometallurgy; 152, 33-43. http://doi.org/10.1016/j.hydromet.2014.12.004

Cox, A. \& Bryan, C.G., (2017). Insights into Heap Bioleaching at the Agglomerate-Scale. Solid State Phenom. Trans. Tech. Publ. 185-188. https://doi.org/10.4028/www.scientific.net/SSP.262.185

Ehrlich, H.L. (2001). Past, present and future of biohydrometallurgy.
Hydrometallurgy; 59, 127-134. https://doi.org/10.1016/S0304386X(00)00165-1

Ewart, D.K. \& Martin, N.H. (1991). The extraction of metals from ores using bacteria. Adv. Inorg. Che; 36, 103-135. https://doi.org/10.1016/ S0898-8838(08)60038-0

Fernandes, J.C. \& Henriques, F.S. (1991). Biochemical, physiologi$\mathrm{cal}$, and structural effects of excess copper in plants. Bot. Rev; 57, 246273. https://doi.org/10.1007/ BF02858564

Giaveno, A., Lavalle, L., Guibal, E. \& Donati, E. (2008). Biological ferrous sulfate oxidation by $A$. ferrooxidansimmobilized on chitosan beads. $J$. Microbiol. Methods; 72, 227-234. https://doi.org/10.1016/j.mimet.2008.01.002

Gupta, A., Dutta, A., Sarkar, J., Paul, D., Panigrahi, M.K. \& Sar, P. (2017). Metagenomic exploration of microbial community in mine tailings of Malanjkhand copper project, India. Genomics Data; 12, 11-13. https://doi.org/10.1016/j.gdata.2017.02.004

Hao, X.D., Liang, Y.L., Yin, H.Q., Ma, L.Y., Xiao, Y.H., Liu, Y.Z., Qiu, G.Z. \& Liu, X.D. (2016). The effect of potential heap construction methods on column bioleaching of copper flotation tailings containing high levels of fines by mixed cultures. Minerals Engineering; 98, 279-285. http://dx.doi.org/10.3390/ $\min 8020032$ 
Harrison, S.T.L. (2016). Biotechnologies that utilize acidophiles. In: Quatrini, R., Johnson, D.B. (Eds.), Acidophiles: Life in Extremely Acidic Environments. Caistor Academic Press, Haverhill, UK, 265-283.

Johnson, D.B. (2014). Biomining - biotechnologies for extracting and recovering metals from ores and waste materials. Curr. Opin. Biotechnol; 30, 24-31. https://doi.org/10.1016/j.copbio.2014.04.008

Karlfeldt, F.K., Yillin, L. \& Strömvall, A.M. (2013). Remediation of metal polluted hotspot areas through enhanced soil washing evaluation of leaching methods. $J$. Environ. Manag; 128, 489-496. https://doi.org/10.1016/j.jenvman.2013.05.056

Kefeni, K.K., Msagati, T.A.M. \& Mamba, B.B. (2017). Acid mine drainage: Prevention, treatment options, and resource recovery: A review. Journal of Cleaner Production; 151, 475-493. http:// dx.doi.org/10.1016\%2Fj.jclepro.2017.03.082

Kondrat'eva, T.F., Pivovarova, T.A., Bulaev, A.G., Melamud, V.S., Muravyov, M.I., Usoltsev, A.V. \& Vasil'ev, E.A. (2012). Percolation bioleaching of copper and zinc and gold recovery from flotation tailings of the sulfide complex ores of the Ural region, Russia. Hydrometallurgy; 111-112, 82-86. http:// dx.doi.org/10.1016/j.hydromet. 2011.10 .007
Korehi, H., Blothe, M. \& Schippers, A. (2014). Microbial diversity at the moderate acidic stage in three different sulfidic mine tailings dumps generating acid mine drainage. Research in Microbiology; 165, 713718. https://doi.org/10.1016/j.resmic.2014.08.007

Maksymiec, W. (1997). Effect of copper on cellular processes in higher plants. Photosynthetica; 34, 321-342. https://doi.org/10.1023/A:1006818815528

Mazuelos, A., Romero, R., Palencia, I., Iglesias, N. \& Carranza, F. (1999). Continuous ferrous iron biooxidation in flooded packed bed reactors. Miner. Eng; 12(5), 559-564.

Mishra, D., Kim, D.J., Ahn, J.G. \& Rhee, Y.H. (2005). Bioleaching: a microbial process of metal recovery; A review. Met. Mater. Int; 11, 249256. https://doi.org/10.1007/ BF03027450

Nejeschlebová, L., Sracek, O., Mihaljevic ${ }^{\vee}$, M., Ettler, V., Kř́bek, B. \& et al. (2015). Geochemistry and potential environmental impact of the mine tailings at Rosh Pinah, southern Namibia. Journal of African Earth Sciences; 105, 17-28. https://doi.org/10.1016/j.jafrearsci.2015.02.005

Park, S.M., Yoo, J.C., Ji, S.W., Yang, J.S. \& Baek, K. (2015). Selective recovery of dissolved $\mathrm{Fe}, \mathrm{Al}$, $\mathrm{Cu}$, and $\mathrm{Zn}$ in acid mine drainage based on modeling to predict precipitation pH. Environ. Sci. Pollut. 
Res; 22, 3013-3022. https://doi. org/10.1016/j.jece.2014.07.021

Rawlings, D.E. (2013). Biomining: Theory, Microbes and Industrial Processes. Springer, Berlin-New York

Simate, G.S. \& Ndlovu, S. (2014). Acid mine drainage: Challenges and opportunities. J. Environ. Chem. Eng; 2, 1785-1803. https://doi. org/10.1016/j.jece.2014.07.021

Vahidi, E. \& Zhao, F. (2016). Life cycle analysis for solvent extraction of rare earth elements from aqueous solutions. In: Rewas 2016. Springer, 113-120. https:// doi.org/10.1007/978-3-31948768.7_17

Volchko, Y., Norrman, J., Rosén, L. \& Karlfeld, F.K. (2017). Cost-ben- efit analysis of copper recovery in remediation projects: A case study from Sweden. Science of the Total Environment, 605-606, 300-314. http://dx.doi.org/10.1016/j.scitotenv.2017.06.128

Watling, H. (2016). Microbiological advances in biohydrometallurgy. Fortschr. Mineral; 6(2), 49. https:// doi.org/10.3390/min6020049

Yin, S., Wang, L., Kabwe, E., Chen, X., Yan, R. \& et al. (2018). Copper Bioleaching in China: Review and Prospect. Minerals; 8(32). 1-26. http://dx.doi.org/10.3390/ $\min 8020032$

Yruela, I. (2005). Copper in plants. Braz. J. Plant Physiol; 17, 145-156. http://dx.doi.org/10.1590/S167704202005000100012 
